\title{
UM PROCESSO DIRIGIDO POR MODELOS PARA PROMOVER O ALINHAMENTO ENTRE A ESTRATÉGIA DE NEGÓCIO E TI
}

\author{
Karlos T. M. V. Oliveira ${ }^{1}$, Geam Carlos de Araújo Filgueira ${ }^{1}$, \\ Jannayna Domingues Barros Filgueira ${ }^{2}$, Emanuel Dantas Filho ${ }^{3}$, Paulo de Figueiredo Pires ${ }^{4}$ \\ ${ }^{1}$ Instituto Federal de Educação, Ciência e Tecnologia do Rio Grande do Norte (IFRN) \\ ${ }^{2}$ Universidade Estadual da Paraíba (UEPB) \\ ${ }^{3}$ Instituto Federal de Educação, Ciência e Tecnologia do Rio Grande do Norte (IFCE) \\ ${ }^{4}$ Universidade Federal do Rio de Janeiro (UFRJ) \\ $<$ karlos.oliveira@ifrn.edu.br>, <geam.filgueira@ifrn.edu.br $>$ \\ <jannayna@uepb.edu.br>, <emanuel.filho@ifce.edu.br>, <paulo.fpires@dcc.ufrj.br>
}

DOI: 10.21439/conexoes.v10i2.771

\begin{abstract}
Resumo. Esse trabalho apresenta uma abordagem baseada em modelos que tem como objetivo propor a criação de um processo de alinhamento estratégico concreto que seja capaz de permitir a rastreabilidade entre os objetivos organizacionais e as atividades dos processos de negócio de uma organização, tomando como base para isso, o formalismo MDA. Esse processo provê o alinhamento através da definição de diferentes níveis de modelos, os quais provêm uma transição continua desde a representação do conhecimento estratégico até a representação operacional das atividades de processos que visam atender a essa estratégia. Para alcançar o objetivo do trabalho será utilizado BSC e GORE para realizar um mapeamento da estratégia da organização em iniciativas de processos de negócios. Em seguida, utilizar transformações baseadas em MDA para obter modelos de processos de negócio rastreáveis expressos em BPMN. Como resultado, foi possível visualizar e rastrear através de diagramas o relacionamento entre a estratégia da empresa e as decisões de cada setor. Em especial, foi observado onde as decisões da área de TI refletem nos objetivos de negócio da empresa.
\end{abstract}

Palavras-chaves: Estratégia. Alinhamento. Processo. Rastreabilidade.

\begin{abstract}
This paper presents a model-based approach that aims to propose the creation of a concrete strategic alignment process that is able to provide traceability between organizational objectives and activities of the business processes of an organization, taking as a basis for this, MDA formalism. This process provides the alignment by setting different levels of models, which come from a transition continues from the representation of knowledge available to the operating activities of representation of processes to meet this strategy. To achieve the objective of this study will be used BSC and GORE to perform an organizational strategy of mapping business process initiatives. Then use changes based on MDA for models traceable business processes expressed in BPMN. As a result, it was possible to visualize and track through diagrams the relationship between the company's strategy and decisions of each sector. In particular, it was observed where the IT department's decisions reflect the business goals of the company.
\end{abstract}

Keywords: Strategy. Alignment. Process. Tracking.

\section{INTRODUÇÃO}

Resultados de várias pesquisas mostram a relevância do alinhamento estratégico tanto para o executivo de negócio, quanto para o executivo de TI (Niederman et al., 1996). De fato, os executivos de TI têm conside- rado o alinhamento estratégico como um dos objetivos principais da área de TI justamente pela possibilidade de identificação de novas oportunidades de negócios e obtenção de vantagens competitivas baseadas em soluções de TI. O BPM (Business Process Management) vem sendo apontado como uma maneira promissora de 
técnica gerencial que tem como base à realização do alinhamento estratégico (HARRINGTON; ESSELING; NIMWEGEN, 1997; SMITH; FINGAR, 2003, HARMON, 2003, JESTON; NELIS, 2006). Além disso, modelos de governança de TI, tal como o COBIT (Control Objectives for Information and related Technology) e o ITIL (Information Technology Infrastructure Library) também possuem como base para sua realização, o alinhamento estratégico (FERNANDES; ABREU, 2008).

É importante atentar para o fato de que os modelos de governança, bem como a técnica gerencial do BPM se preocupam bem mais do que apenas o alinhamento estratégico; na verdade, esse alinhamento estratégico é apenas um subconjunto de tudo o que esses modelos são capazes de oferecer, porém, o alinhamento acaba sendo a peça chave para que todo o modelo funcione corretamente.

Existem também diversos modelos conceituais centrados, único e exclusivamente no alinhamento estratégico, dentre alguns dos existentes podemos citar o modelo de Venkatraman (1993), o modelo de Brodbeck (2001) e o modelo de Reich (1992). O modelo definido por Henderson e Venkatraman encontra-se em um alto nível de abstração, ou seja, não foca na operacionalização do alinhamento. O modelo de Brodbeck surge para sanar esse problema, propondo mecanismos que visam prover um alinhamento contínuo entre o negócio e TI. Porém, a proposta carrega um alto grau de complexidade derivado da inclusão de novos elementos e conceitos ao modelo proposto por Henderson e Venkatraman bem como novos elementos para modelagem informacional para representar as operações, objetivos e estratégias do negócio. Essa complexidade dificulta a implementação do modelo de Brodbeck de forma integral. Já o modelo de Reich não leva em consideração a interatividade entre medidas financeiras e não-financeiras. Observa-se também que poucos modelos de alinhamento existentes na literatura tratam do problema de rastreabilidade entre TI e a estratégia de negócio. A rastreabilidade é necessária, por exemplo, quando se deseja saber exatamente qual meta ou plano de ação definida na estratégia de negócio não foi ou deixou de ser aceita devido a uma alteração no plano de TI.

Uma recente pesquisa de mercado feita com 164 organizações dos EUA e UK que usam algum tipo de modelo como premissa ao alinhamento estratégico mostra que mesmo apesar de todo o investimento que as empresas vêm fazendo nos últimos anos apenas $37 \%$ afirmaram obter melhorias claras e mensuráveis, $42 \%$ responderam ainda não obterem clareza em seus mapeamentos, $18 \%$ responderam não ter obtido resultado nenhum e $3 \%$ não souberam medir seus resultados Vol- mer (2007). Esses números vislumbram bem que ainda existe uma carência real na obtenção do alinhamento; até pelo fato de não existir um processo concreto que mostre os passos necessários que uma organização deve fazer para se obter esse objetivo. Na verdade o que existe são conceitos e esquemas teóricos, que a partir deles e das experiências das pessoas que já operam esses modelos direcionam a organização para o caminho correto (BALDAM et al., 2007). Ocasionando, portanto, em prováveis problemas de comunicação, perda de informação ou de rastreamento. Nesse contexto, a motivação e justificativa desse trabalho, fundamentada no conceito de alinhamento estratégico entre os planos de negócio e TI, está na busca de uma solução plausível que ajude a diminuir de fato o gap ainda existente entre esses dois mundos e consequentemente tornar todo o seu mapeamento rastreável.

Com base nos problemas ainda em aberto, o objetivo desse trabalho é propor a criação de um processo de alinhamento estratégico concreto voltado mais para a parte prática do que a parte conceitual, a qual seja capaz de permitir a rastreabilidade entre os objetivos organizacionais e as atividades dos processos de negócio, tomando como base para isso uma abordagem orientada a modelos que forneça mecanismos suficientes para suprir essas necessidades, como por exemplo, o formalismo MDA (Model Driven Architecture) (OMG, 2001). Para que seja possível atingir esse objetivo, a seguinte metodologia foi adotada. O primeiro passo consisti em efetuar um mapeamento prévio da estratégia da organização (sua visão e missão) em iniciativas de processo, que são os planos de ação requeridos pela organização para alcançar o alinhamento almejado. Nesse primeiro momento será usado o BSC (Balanced Scorecard), que é uma ferramenta de gestão estratégica cuja característica é traduzir a estratégia da empresa em termos operacionais. Tendo o plano de ação em mãos, o segundo passo consiste em explicitar as razões e motivações associadas ao que se espera desse plano. Isso significa analisar elementos de processos que permitam representar todos os aspectos relevantes que fundamentam o "porquê" daquilo que se pretende representar. A engenharia de requisitos orientada a objetivos (GORE - Goal Oriented Requirements Engineering) tem tido um papel fundamental para apontar estratégias e técnicas que permitam representar melhor estes "porquês" e consequentemente servir como ponte (rastreabilidade) para se chegar ao processo de negócio (ROLLAND; SOUVEYET; ACHOUR, 1998). Por fim, com um modelo GORE a disposição, o último passo consiste em fazer transformações baseadas no formalismo MDA que podem ser definidas a fim de se obter de forma semi-automatizada 
modelos de processos de negócio rastreáveis expressos em BPMN (Business Process Modeling Notation), notação padrão para modelagem dos processos. Na seção seguinte será detalhado melhor cada uma dessas ferramentas e tecnologias.

\section{MATERIAL E MÉTODOS}

Para o desenvolvimento deste trabalho, foram utilizadas diferentes abordagens e tecnologias tais como: $B a$ lanced Scorecard (BSC) (KAPLAN; NORTON, 2004), técnica utilizada para fazer o mapeamento da estratégia da organização em planos de ações a serem seguidos; Knowledge Acquisition in automated Specification (KAOS) (Lamsweerde e Letier, 2003), abordagem da engenharia de requisitos orientada a objetivos (GORE) que permite representar a forma com que os planos de ações devem ser tratados; Model Driven Architecture (MDA) (OMG, 2006), abordagem de desenvolvimento orientado a modelos (MDD) usado para formalizar os mapeamentos entre o mundo dos negócios e o mundo da TI; e Business Process Modeling Notation (BPMN) (BPMN, 2004), notação padrão usada para representar os processos de negócio derivados dos objetivos estratégicos.

BSC é uma técnica de gestão organizacional cuja finalidade é traduzir a estratégia de negócio de uma organização em planos de ação através de um mapa estratégico organizado em quatro perspectivas (KAPLAN; NORTON 1997): (i) financeira - representa questões de lucratividade da organização; (ii) cliente - relacionada a satisfação do cliente com relação ao produto e/ou serviço provido pela organização; (iii) processos internos - processos críticos nos quais as organizações devem alcançar a excelência, e (iv) inovação \& aprendizado - objetivos e indicadores que servem como base de desempenho futuro da organização. Por exemplo, nessa perspectiva pode-se englobar o nível de satisfação dos empregados de acordo com o clima de trabalho.

KAOS é uma abordagem da engenharia de requisitos orientada a objetivos que fornece uma linguagem formal rica para a captura de requisitos funcionais, nãofuncionais e organizacionais, provendo facilidades para a aquisição/elaboração de requisitos a qual são representadas como objetivos (goals). KAOS é descrito em (Lamsweerde e Letier 2003) como um framework multiparadigma que permite combinar diferentes níveis de expressão e raciocínio: (i) semi-formal, para modelagem e estruturação dos objetivos; e (ii) formal, para uma maior precisão de raciocínio, como por exemplo, o controle de sistemas críticos que envolvem riscos à vida do usuário (controle aéreo).

BPMN é uma especificação criada pela BPMN
(2004) que permite a representação gráfica de processos de negócio. Seu principal objetivo é suportar a modelagem através de uma notação simples e poderosa que ofereça facilidades para especificação e modelagem dos processos pelos analistas de processos.

MDA é uma abordagem baseada na ideia de que modelos são os artefatos mais importantes dentro do desenvolvimento de sistemas, e cujo objetivo é separar decisões orientadas ao negócio de decisões relativas a plataformas computacionais, permitindo assim maior flexibilidade durante as fases de especificação e de desenvolvimento de sistemas (FRANKEL, 2003). Para atingir esse objetivo, um processo MDA define modelos com diferentes níveis de abstração: (i) CIM (Computation Idependent Model); (ii) PIM (Platform Independent Model); e (iii) PSM (Platform Specific Model). O CIM é o modelo de mais alto nível, representando as restrições do sistema e funcionalidades que não dependem de modelo computacional. Muitas vezes chamado de modelo de domínio ou modelo de negócio, ele é útil tanto para ajudar o analista a compreender o problema quanto para servir como fonte de vocabulário a ser usado em outros modelos. O PIM descreve o sistema incluindo componentes necessários à sua implantação em um sistema computacional, porém não apresenta os detalhes da tecnologia que será usada na implementação. O PSM é responsável por combinar a especificação do modelo PIM com detalhes de uma determinada plataforma, incluindo informações como serviços específicos de uma determinada plataforma que vai ser usada para implementar as funções do sistema.

\section{RESULTADOS E DISCUSSÃO}

\subsection{O Processo de Alinhamento}

O processo de alinhamento proposto nesse trabalho é baseado na abordagem MDA. Como nosso foco são modelos completamente independentes de computação (orientado a negócio), o processo proposto se concentra na camada CIM. O processo de alinhamento proposto organiza o CIM em três camadas: (i) CIM-0: representa a estratégia de negócio e suas iniciativas (planos de ação), sendo especificado por mapas estratégicos de acordo com o BSC; (ii) CIM-1: responsável por representar o refinamento para cada iniciativa existente no modelo anterior, sendo especificado com o uso do KAOS; e (iii) CIM-2: responsável pela representação dos processos de negócio da organização alinhados com sua estratégia.

O mapeamento entre o CIM-0 e CIM-1 é feito de forma manual, isso ocorre devido a sua complexidade e ao elevado grau de raciocínio requerido, já que mui- 
tas variáveis estão envolvidas (por exemplo: ambiente, clientes, fornecedores, políticas da organização e etc), tornando a elaboração desse modelo por meios automatizados extremamente cara e sem garantia de corretude. Já o mapeamento entre o CIM-1 e CIM-2 é automatizado, usando para isso transformações MDA; tal automatização só é possível graças à riqueza de informações contida nos modelos KAOS. Existe também outro mapeamento automatizado, que é realizado entre o mapa estratégico da organização e sua tabela complementar, a qual é realizada antes mesmo de se obter o modelo CIM-1 presente na primeira fase do processo (detalhes serão vistos nas seções a seguir). O processo de alinhamento proposto é apresentado na Figura 1

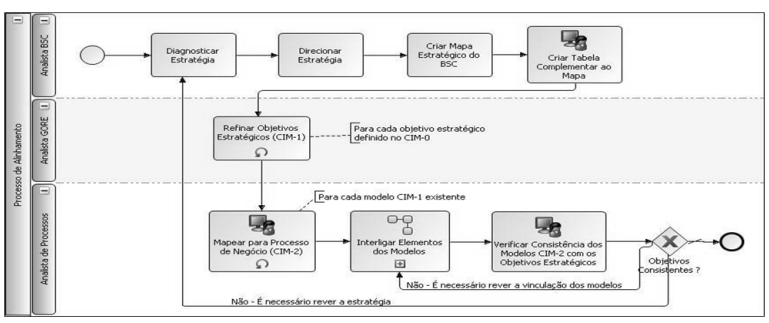

Figura 1: Processo de Alinhamento Proposto.

O processo ilustrado na Figura 1 apresenta uma subdivisão em swimlanes, onde cada swimlane pode ser interpretada como uma fase do processo, executada por um dos três atores do sistema. O ator "Analista BSC" é um Analista de Negócios com especialidade em BSC, o ator "Analista GORE" é um analista com especialidade em KAOS e o "Analista de Processos" é um analista com especialidade em BPMN. A primeira fase do processo é contida de quatro atividades distintas, onde, após criar-se a estratégia da organização (união das três primeiras atividades) é necessário definir suas iniciativas, em outras palavras, seu plano de ação (última atividade dessa fase). Na segunda fase do processo, existe apenas uma atividade, que é refinar as iniciativas existentes para cada objetivo estratégico modelado na fase anterior. Por fim é na terceira fase que se é derivado o modelo de processos de negócio, interligado com elementos do modelo - atividade de "Interligar Elementos dos Modelos" a qual consiste de um sub-processo que descreve como o analista de processos deve atuar para vincular os elementos do modelo CIM-0 com os elementos do modelo CIM-2 - e finalmente verificar se os objetivos estão consistentes, ou seja, se está alinhado com a estratégia de negócio. Ainda na Figura 1 , podese observar que existem atividades sendo representadas com um ícone de um computador em seu centro. Esses tipos de atividades são do tipo User Task, o que significa dizer que serão realizadas por intermédio de um software.

\subsection{Primeira Fase do Processo}

Essa fase é de responsabilidade do Analista BSC e engloba as atividades de "Diagnosticar Estratégia", "Direcionar Estratégia", "Criar Mapa Estratégico do BSC" e "Criar Tabela Complementar ao Mapa". A primeira atividade consiste em analisar o estado atual da estratégia da organização, visando sua situação competitiva no mercado e práticas adotadas. Serve como base para o bom entendimento e direcionamento da estratégia. $\mathrm{Na}$ segunda atividade, Direcionar Estratégia, a organização toma uma decisão de orientação para sua estratégia com o intuito de atingir futuros objetivos. A terceira atividade, Criar Mapa Estratégico é onde se concentra de fato detalhes da estratégia escolhida. Nela o analista deve, além de explicitar a missão e visão da organização, alocar os objetivos estratégicos nas quatro perspectivas existentes do BSC, correlacionando-as entre si numa relação de causa e efeito.

Nesse processo poderão ou não surgir lacunas (gaps) no inter-relacionamento, que deverão ser eliminadas ou preenchidas a partir de discussões e análises da estratégia da organização. A construção desse mapa estratégico é uma atividade totalmente manual. A Figura 2 apresenta um exemplo fictício de um mapa estratégico de uma empresa de varejo.

A construção do mapa estratégico inicia com a definição da visão e missão da organização. Os objetivos estratégicos de cada perspectiva são posteriormente definidos. Na perspectiva financeira da Figura 2, estão envolvidos três objetivos estratégicos: Aumentar lucros, Incrementar participação de mercado e Disponibilizar preços mais acessíveis que a concorrência. Tanto o objetivo "Incrementar participação de mercado", quanto o objetivo "Disponibilizar preços mais acessíveis que a concorrência" surte efeito no objetivo "Aumentar lucros' (relacionamento causa-efeito), que por sua vez, é o objetivo que representa o crescimento financeiro da organização. Porém, o sucesso financeiro e a realização da missão são uma consequência natural do sucesso no mercado, que pode ser alcançado através de um bom relacionamento com os clientes. Dessa forma, a perspectiva do cliente enfatiza a importância de aumentar o grau de satisfação do cliente para causar efeito no objetivo descrito na perspectiva financeira. Todavia, o sucesso no mercado, e, por conseguinte, o crescimento financeiro, apenas será conquistado a partir da excelência nos processos internos estratégicos. Isso é especificado no mapa pelos objetivos "Oferecer segurança no serviço 
prestado" e "Oferecer eficiência na gestão de estoque". Finalmente, na raiz do mapa, encontra-se a perspectiva Inovação \& Aprendizado, com os objetivos de satisfação e capacitação dos empregados, que deverão assegurar a continuidade de aprendizado e desenvolvimento da organização.

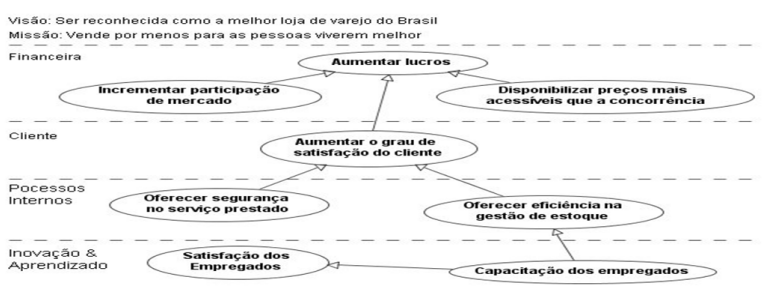

Figura 2: Mapa Estratégico de uma Empresa Fictícia de Varejo.

A fim de complementar o mapa estratégico e concretizar a construção do modelo CIM-0, o último passo realizado pelo analista BSC é a atividade de "Criar tabela complementar ao mapa (CIM-0)". Nessa atividade o analista BSC deve preocupar-se com a definição dos indicadores, metas e iniciativas responsáveis para implementação da estratégia estabelecida.

A finalidade dessa atividade é facilitar o elo entre os elementos do mapa estratégico e os refinamentos a serem feitos pelo analista GORE (refinamento das iniciativas). A Tabela 1 apresenta a saída dessa atividade.

A coluna intitulada "Ef" (Efeito) serve para informar textualmente o relacionamento existente entre os objetivos estratégicos ilustrados na Tabela 11 Como exemplo temos que o objetivo "Incrementar participação de mercado" (item 2, Tabela 1) causa um efeito no objetivo 1 (Aumentar lucros); implicando dizer que havendo um aumento de participação da empresa no mercado, consequentemente haverá um aumento nos lucros. Já a coluna "Vl" (Valor), especifica a influência de um objetivo sobre outro. Por exemplo: o objetivo "Oferecer segurança no serviço prestado" é responsável por 30\% do êxito do objetivo "Aumentar o grau de satisfação dos clientes".

Essa atividade é do tipo User Task, e sua realização são feita através de uma ferramenta de suporte. Isso significa dizer que existem regras de mapeamento escritas em ATL (Atlas Definition Language) (ATLAS, 2012) com seus respectivos metamodelos no intuito de formalizar as informações contidas na Figura 2 para Tabela 1 As Figuras 3 e 4 ilustram esses metamodelos.

De acordo com o metadamodelo da Figura 3, um mapa estratégico consiste de quatro perspectivas distintas, cada qual podendo ser do tipo "Financeira", "Cliente", "Processos Internos" e "Inovação e Aprendi-

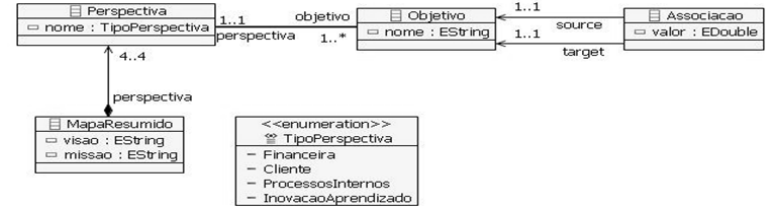

Figura 3: Metamodelo Referente ao Mapa Estratégico.

zado”. Cada perspectiva é constituída de um ou vários objetivos e cada objetivo pode estar associado a outro objetivo, porém, devendo estar detido de um valor percentual que informe o quanto cada um influencia o outro.

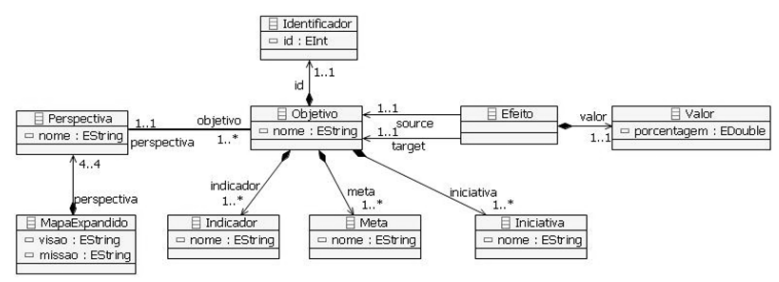

Figura 4: Metamodelo Referente à Tabela Complementar ao Mapa Estratégico.

Em adicional ao metamodelo do mapa resumido, o metamodelo da Figura 4 é constituído de mais quatro elementos, são eles: Indicador, Meta, Iniciativa e Identificador, esse último é utilizado para identificar os objetivos na tabela. $\mathrm{O}$ antigo elemento chamado "Associação" passou a ser chamado de "Efeito" e contém uma composição com o elemento "Valor". Essa mudança na nomenclatura foi feita para melhor manuseio com o modelo transformado.

\subsection{Segunda Fase do Processo}

Na segunda fase do processo entra em cena o Analista GORE, que tem a responsabilidade de refinar as iniciativas associadas aos objetivos estratégicos no intuito de evidenciar como esses objetivos deverão ser alcançados. Como resultado tem-se a criação do modelo orientado a objetivos (CIM-1). Como dito anteriormente, essa atividade é feita de forma manual devido a sua complexidade e pela existência de diversas variáveis que inviabilizam sua automatização. A Figura 5 ilustra um exemplo de como ficaria o CIM-1 após o refinamento da iniciativa "Efetuar venda eficiente" referente ao objetivo estratégico "Aumentar o grau de satisfação do cliente" ilustrado na Tabela 1

Cada paralelograma da Figura 5 representa um goal ou subgoal, diferenciados conceitualmente apenas pela 
Tabela 1: Dados complementares ao Mapa Estratégico.

\begin{tabular}{|c|c|c|c|c|c|c|c|}
\hline $\begin{array}{l}\text { Perspe } \\
\text { ctiva }\end{array}$ & & $\begin{array}{c}\text { Obj. } \\
\text { Estratégico }\end{array}$ & Ef & V1 & Indicadores & Metas & Iniciativas \\
\hline \multirow{3}{*}{ 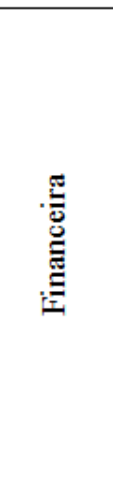 } & 1 & $\begin{array}{l}\text { Aumentar os } \\
\text { Lucros }\end{array}$ & - & - & $\begin{array}{l}\text { Porcentagem de } \\
\text { crescimento do } \\
\text { faturamento líquido }\end{array}$ & $\begin{array}{l}\text { Aumentar } 15 \% \\
\text { em relação ao } \\
\text { ano anterior }\end{array}$ & $\begin{array}{l}\text { Oferecer promoção do } \\
\text { serviço oferecido. } \\
\text { Ampliar mix de } \\
\text { produtos }\end{array}$ \\
\hline & 2 & $\begin{array}{l}\text { Incrementar } \\
\text { participação } \\
\text { de mercado }\end{array}$ & 1 & $25 \%$ & $\begin{array}{l}\text { Porcentagem de } \\
\text { participação de } \\
\text { mercado }\end{array}$ & $\begin{array}{c}\text { Estar entre os } \\
\text { cinco primeiros } \\
\text { do Brasil }\end{array}$ & $\begin{array}{c}\text { Intensificar divulgação } \\
\text { dos produtos }\end{array}$ \\
\hline & 3 & $\begin{array}{c}\text { Dispor } \\
\text { preços mais } \\
\text { acessíveis } \\
\text { que a } \\
\text { concorrência }\end{array}$ & 1 & $50 \%$ & $\begin{array}{l}\text { Preço total da } \\
\text { venda }\end{array}$ & $\begin{array}{l}\text { Reduzir em } 5 \% \\
\text { o valor total da } \\
\text { venda }\end{array}$ & Reduzir despesas \\
\hline : & 4 & $\begin{array}{l}\text { Aumentar o } \\
\text { grau de } \\
\text { satisfação } \\
\text { dos clientes }\end{array}$ & 1 & $60 \%$ & $\begin{array}{l}\text { Porcentagem de } \\
\text { satisfação }\end{array}$ & $\begin{array}{c}90 \% \text { dos } \\
\text { clientes } \\
\text { satisfeitos com a } \\
\text { comodidade do } \\
\text { serviço } \\
\text { prestados \& } \\
100 \% \text { dos } \\
\text { clientes } \\
\text { satisfeitos com } \\
\text { o tempo médio } \\
\text { da compra }\end{array}$ & $\begin{array}{c}\text { Efetuar venda } \\
\text { eficiente. Manter } \\
\text { estoque sob controle }\end{array}$ \\
\hline \multirow{2}{*}{ 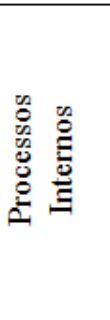 } & 5 & $\begin{array}{c}\text { Oferecer } \\
\text { segurança no } \\
\text { serviço } \\
\text { prestado } \\
\end{array}$ & 4 & $30 \%$ & $\begin{array}{l}\text { Porcentagem de } \\
\text { furtos }\end{array}$ & $\begin{array}{l}\text { Reduzir a zero o } \\
\text { número de } \\
\text { furtos no } \\
\text { estabelecimento }\end{array}$ & $\begin{array}{c}\text { Implantar política de } \\
\text { segurança }\end{array}$ \\
\hline & 6 & $\begin{array}{l}\text { Oferecer } \\
\text { eficiência na } \\
\text { gestão de } \\
\text { estoque }\end{array}$ & 4 & $40 \%$ & $\begin{array}{c}\text { Tempo de aquisição } \\
\text { e organização do } \\
\text { estoque }\end{array}$ & $\begin{array}{l}\text { Nunca deixar } \\
\text { que um produto } \\
\text { falte para o } \\
\text { cliente }\end{array}$ & $\begin{array}{l}\text { Implantar política de } \\
\text { logística. Requisitar } \\
\text { produtos para estoque }\end{array}$ \\
\hline \multirow{2}{*}{ 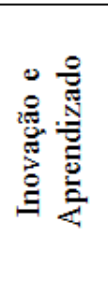 } & 7 & $\begin{array}{l}\text { Satisfação } \\
\text { dos } \\
\text { empregados }\end{array}$ & - & - & $\begin{array}{c}\text { Índice dos } \\
\text { empregados } \\
\text { satisfeitos } \\
\end{array}$ & $\begin{array}{c}95 \% \text { dos } \\
\text { empregados } \\
\text { satisfeitos } \\
\end{array}$ & $\begin{array}{l}\text { Implantação de } \\
\text { programação de } \\
\text { incentivo }\end{array}$ \\
\hline & 8 & $\begin{array}{l}\text { Capacitação } \\
\text { de } \\
\text { empregados }\end{array}$ & $\begin{array}{l}6 ; \\
7\end{array}$ & $\begin{array}{c}20 \% \\
15 \%\end{array}$ & $\begin{array}{c}\text { Número de } \\
\text { capacitações ao ano }\end{array}$ & $\begin{array}{c}\text { Capacitação } \\
\text { trimestral } 95 \% \\
\text { dos empregados } \\
\text { para o cargo }\end{array}$ & $\begin{array}{c}\text { Criação de cronograma } \\
\text { de capacitações e } \\
\text { avaliações }\end{array}$ \\
\hline
\end{tabular}


sua localização na árvore. Por exemplo: o paralelograma que se encontra na raiz da figura é um goal, enquanto os seus nodos filhos, são subgoals. Os paralelogramas caracterizados por bordas em negrito são considerados os requisitos e não podem mais ser refinados.

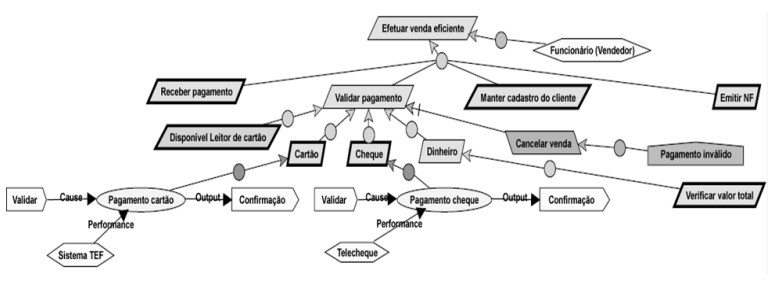

Figura 5: Refinamento da Iniciativa "Efetuar venda eficiente".

É importante salientar que o Analista GORE deve efetuar o refinamento até um ponto em que o mesmo perceba que o objetivo chegou a um nível terminal, ou seja, que não necessite mais ser refinado, já que se encontra pronto para ser operacionalizado (transformado para processo). Existem dois tipos de refinamento: AND-refinement e OR-refinement. O AND-refinement relaciona uma meta a um conjunto de sub-metas, isso significa que a única condição suficiente para satisfazer a meta é satisfazer todas as sub-metas. O OR-refinement relaciona uma meta a um conjunto de refinamentos alternativos, isso significa dizer que satisfazer um dos refinamentos é condição suficiente para satisfazer a meta. Graficamente, o que diferencia um refinamento do tipo AND de um refinamento do tipo OR, são as disposições de suas setas. Por exemplo, o goal "Efetuar venda eficiente" tem um refinamento do tipo AND contendo: "Receber pagamento", "Validar pagamento", "Manter cadastro de clientes" e "Emitir NF" devido ao fato de ambas as setas estarem ligadas ao mesmo círculo. Já no caso do refinamento existente para o subgoal "Validar pagamento", pode-se perceber que cada elemento filho está ligando independentemente com o seu elemento pai.

O goal "Cancelar Venda", é na verdade um obstáculo existente no ambiente; que caso venha a ocorrer, irá prejudicar a obtenção de sucesso do subgoal "Validar pagamento" e consequentemente, o goal "Efetuar venda eficiente". É aconselhável, sempre que se usar o conceito de obstáculo (comportamento indesejável), fazer logo em seguida uso do conceito de propriedade de domínio, que tem a finalidade de esclarecer o que se espera de fato para que esse comportamento indesejável não ocorra. Os agentes (representados por losangos) podem ser vistos como os responsáveis pela realização de um determinado goal, subgoal ou requisito. Quando associado a uma operação (representado por uma elipse) juntamente com seus eventos de entrada e saída, significa dizer que o goal/subgoal ou requisito ao qual esse agente está associado está sob responsabilidade de terceiros. Por fim, as especulações (paralelogramas) são usadas sobre goals para informar que é desejado ter algum artefato disponível como pré-requisito para execução do goal.

\subsection{Terceira Fase do Processo}

Essa fase é composta de três atividades, todas sob a responsabilidade do analista de processos, são elas: (i) Mapear para Processo de Negócio; (ii) Interligar Elementos dos Modelos; e (iii) Verificar Consistência dos Modelos CIM-2 com os Objetivos Estratégicos. Na primeira atividade, Mapear para Processo de Negócio (atividade do tipo User Task), o analista faz uso de padrões de mapeamento (transformações MDA) para traduzir a informação contida no modelo orientado a objetivos (CIM-1) criado na fase anterior, em um modelo de processo de negócio (CIM-2). Os padrões utilizados na transformação MDA são:

- O nodo raiz especificado no modelo de origem será definido como o processo de negócio a ser detalhado;

- Para cada subgoal definido no modelo de origem, serão criados sub-processos de mesmo nome no modelo de destino;

- Atividades simples, do tipo tarefa, serão criadas para cada nodo do tipo requisito encontradas no modelo de origem;

- Agentes associados a goals, subgoals e/ou requisitos, serão definidos como swinlanes;

- Agentes associados a operações serão definidos como novos participantes em um novo pool (conceito existente na notação BPMN para representar participantes do processo);

- Eventos serão representados por fluxos de mensagem entre os diferentes participantes;

- Para cada especulação encontrada no modelo de origem, "Data Objects" serão criados para sua representação;

- Serão criados fluxos de sequência entre as atividades simples ou compostas para cada refinamento dos goals existente no modelo de origem;

- Refinamentos de um goal do tipo AND serão tratados como fluxos sequenciais ou gateways paralelos;

Conex. Ci. e Tecnol. Fortaleza/CE, v. 10, n. 2, p. 110 - 120, jul. 2016 
- Refinamentos de um goal do tipo $O R$ serão tratados como gateways inclusivos. Porém, caso estejam associados a propriedades de domínio, serão tratados como gateways exclusivos;

- Obstáculos serão representados como atividades; todavia, estarão sempre associados a gateways exclusivos;

- Questionamentos existentes nos gateways exclusivos, como: "Boa Infa-estrutura?", "Funcionamento correto?", serão criados para cada propriedade de domínio encontrada no modelo de origem. Isso significa dizer que, sempre que existir um obstáculo, uma propriedade de domínio virá logo em seguida a fim de definir seu questionamento.

Assim como a transformação descrita na primeira fase do processo (entre o mapa estratégico e sua tabela complementar) todas as regras citadas acima foram implementadas usando a linguagem de transformação ATL, sendo necessária também a criação dos seus respectivos metamodelos (KAOS e BPMN). As Figuras 6 e 7 ilustram esses metamodelos.

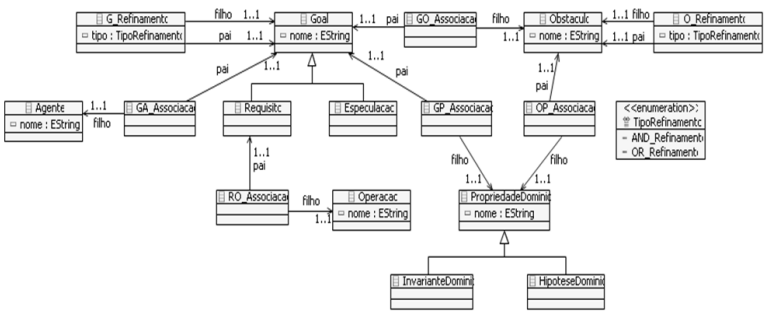

Figura 6: Metamodelo KAOS

Um Goal é um objetivo de alto nível podendo ser especializado em Requisitos e Especulações. O Goal está associado a Agentes, Propriedades de Domínio e Obstáculos, podendo ser refinado até chegar um nível de boa compreensão. Ao efetuar seu refinamento, é necessário especificar o seu tipo: AND-refinament ou $O R$ refinament. Os obstáculos assim como os Goals também podem ser refinados e associados a Propriedades de Domínio. Os requisitos estão associados a operações e as Propriedades de domínio são especializadas em "Invariante de Domínio" e "Hipótese de Domínio".

Um processo consiste de uma ou mais atividades organizadas em zero ou várias swimlanes (Pool e Lanes). A atividade é a parte principal de uma modelagem BPMN e é especializado através de subprocessos e tarefas. As atividades são conectadas entre si através de fluxos; sejam eles fluxos de mensagem ou fluxos de

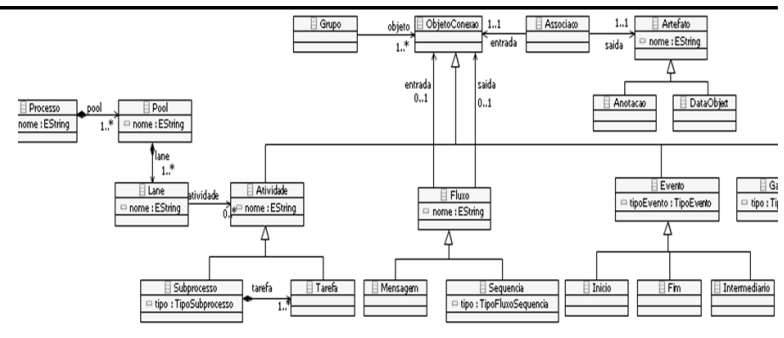

Figura 7: Metamodelo BPMN.

sequência. O fluxo de sequência pode está associado a zero ou um gateway (usado para controlar a convergência e divergência de um fluxo) e a zero ou um evento (que significa que alguma coisa ocorreu durante a execução de um processo de negócio, como por exemplo: o processo iniciou, ou o processo terminou); e por fim, o fluxo de mensagem; que se torna responsável por conectar as atividades distribuídas em diferentes swinlanes.

Além da aplicação da transformação, é dever do analista de processos refinar o novo modelo gerado (se necessário) de acordo com o seu objetivo. A Figura 8 ilustra o mapeamento realizado para o modelo CIM-1 ilustrado na Figura 5

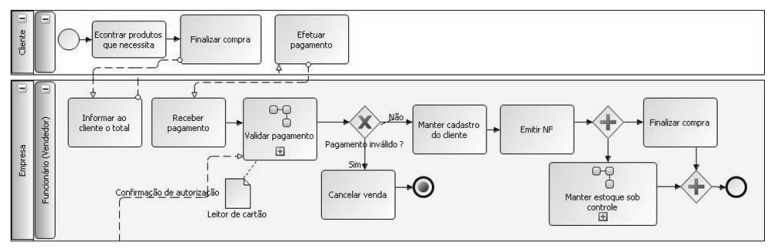

Figura 8: Processo de Negócio "Efetuar venda eficiente".

Após o mapeamento do processo de negócio (Figura 8 ), pode-se observar que o goal raiz passou a ser tratado como o processo de negócio a ser trabalhado; o obstáculo, juntamente com sua propriedade de domínio, transformou-se em tarefa e gateway condicional respectivamente; a especulação em Data Object; subgoals e refinamentos foram representados através de subprocessos e tarefas; e por fim, os agentes passaram a ser representados como participantes do processo. É importante ressaltar que tanto o agente "Inst. financeira" quanto o agente "Telecheque" foram representados como elementos "pool" separados. Isso se deve ao fato do próprio processo "Efetuar venda eficiente" necessitar de ações que são realizadas fora do âmbito da organização (por terceiros), cujas atividades não podem ser gerenciadas (o agente Telecheque foi omitido nessa 
figura por questões de visibilidade). Ainda na Figura 8 percebe-se que durante o mapeamento foram adicionados elementos de detalhamento, como foi o caso de um novo "pool" denominado "Cliente" para representar as atividades designadas por um cliente durante a execução do processo. Outro detalhamento são as referências de dependência entre processos (retângulos representado com uma cruz ao centro), como é o caso do processo "Manter estoque sob controle".

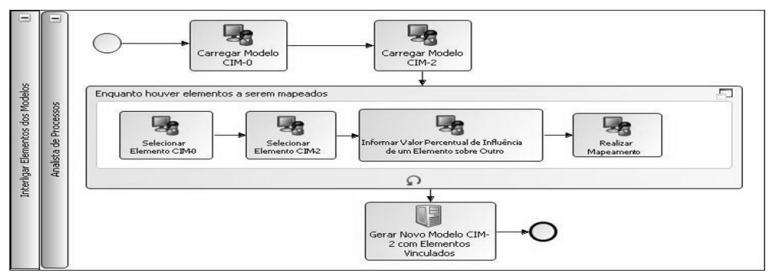

Figura 9: Processo de Rastreamento dos Elementos dos Modelos.

Com a conclusão da atividade Mapear para Processo de Negócio, segue-se a atividade Interligar Elementos dos Modelos, com finalidade de explicitar quais elementos do modelo CIM-2 estão relacionados com quais metas do modelo CIM-0. Ao explicitar esse relacionamento, um valor percentual que indica o quanto cada elemento rastreado está influenciando de forma positiva ou negativa para se atingir uma determinada meta do modelo CIM-0 deve ser informado. A Figura 9 ilustra o diagrama de processos referente ao rastreamento dos modelos. Como pode ser visto, esse processo é realizado em sua totalidade por meio de um conjunto de transformações MDA que, recebendo com entradas um modelo CIM-0, um modelo CIM-1 e posteriormente um mapeamento entre seus elementos (atividade de interligação entre os modelos), produz um novo modelo CIM-2 contendo as referências indicadas aos elementos do CIM-0 (rastreabilidade).

A Figura 10 mostra um exemplo de integração entre o CIM-2 (Figura 9) e o CIM-0 (Tabela 1). Por exemplo, informar que o sub-processo "Manter estoque sob controle" influencia em $40 \%$ para se atingir a meta "90\% dos clientes satisfeitos com a comodidade do serviço prestado (compra)" do objetivo estratégico "Aumentar o grau de satisfação dos clientes". Os valores percentuais são utilizados no presente trabalho para posterior verificação da consistência dos processos de negócio com os objetivos estratégicos (atividade existente na terceira fase do processo), no sentido de informar que processos influenciam ou deixam de influenciar de forma positiva ou negativa na obtenção de uma meta.
$\mathrm{Na}$ Figura 10 valores percentuais que indicam relacionamento de forma positiva entre os elementos dos diferentes modelos são representadas por anotações identificadas pelo padrão "Atinge", que informa o poder de influência (valor percentual) que os elementos do CIM2 representam sobre as metas do CIM-0. Influências negativas não se resumem apenas à metas de objetivos específicos, como é o caso da influência positiva, e sim à atividades, grupos, gateways condicionais e até a processos completos. A Figura 10 apresenta uma dessas influências negativas com o uso do padrão "Contradiz", representada sobre uma anotação na atividade "Cancelar Venda"; que caso venha a ocorrer, irá contradizer em $100 \%$ a meta " $90 \%$ dos clientes satisfeitos com a comodidade do serviço prestado (compra)", representada pelo numeral 1.

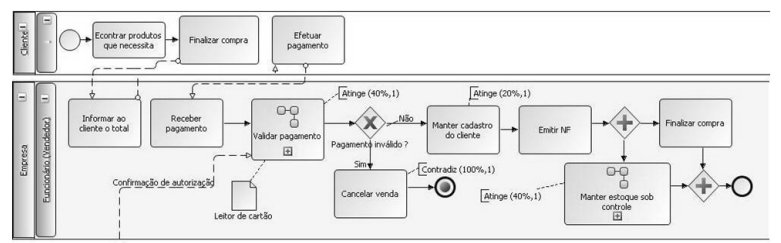

Figura 10: Processo de Negócio "Efetuar venda eficiente" vinculado.

É importante notar que, quando se referenciam outros processos, existe o risco de que uma possível tomada de decisão em um desses processos referenciados (ou até mesmo uma alteração na referência de uma referência) cause um impacto em cascata sobre todos os outros processos. Por exemplo: o modelo "Efetuar venda eficiente" da Figura 6 faz referência ao processo "Manter estoque sob controle", que por sua vez, faz referência a outros dois processos. Esses dois últimos processos podem referenciar outro processo e assim por diante. Isso significa dizer que, qualquer alteração existente em algum desses processos, pode causar diferentes impactos sobre os processos de perspectiva superior. É nesse contexto que entra em cena a última atividade do processo de alinhamento: Verificar Consistência com os Objetivos Estratégicos. Essa atividade tem como finalidade explicitar para o analista de negócio quais objetivos estratégicos foram ou deixaram de ser atingidos devido a essas alterações. Essa atividade de verificação de consistência é uma atividade automatizada e é apresentada nesse trabalho como um serviço oferecido por uma ferramenta de suporte ao processo de alinhamento. Ao seu término, o analista estará apto para tomar qualquer decisão de TI que esteja alinhada ao negócio, por exemplo: "Para obter-se a missão e visão de organização e acima de tudo estando focado no au- 
mento dos lucros pertencentes à perspectiva financeira precisaremos investir em cartões fidelidades e leitores de cartão de crédito para facilitar o pagamento dos clientes. Precisamos implantar também uma política de logística, a qual por meio de algoritmos computacionais seja possível para a organização saber com antecedência quais produtos são mais vendidos e em que período do ano. Investir em tecnologias de Datawarehouse para o armazenamento maciço de informações dos clientes, seus produtos preferidos e tendências de vendas. Será necessário contratar mais três funcionários para trabalhar na área de suporte dos computadores com especialização em Banco de Dados. Precisaremos de um prazo de 10 meses para todas essas medidas entrarem em vigor. Nosso investimento será de $\mathrm{R} \$ 70.000,00$ e a previsão para o retorno do investimento é de 16 meses".

\section{CONCLUSÕES}

Este trabalho surge com uma proposta de um processo de alinhamento estratégico concreto e rastreável que toma como base para sua realização o formalismo MDA além do apoio de outras abordagens amplamente aceitas pelo mercado e pela literatura especializada, tais como: BSC, KAOS e BPMN. Esse processo provê o alinhamento através da definição de diferentes níveis de modelos, os quais provêm uma transição continua desde a representação do conhecimento estratégico de uma organização até a representação operacional das atividades de processos que visam atender a essa estratégia. $\mathrm{O}$ emprego de transformações MDA formaliza o processo de refinamento e prove mecanismos para garantir a rastreabilidade dos elementos nos diferentes níveis de abstração.

Ao propor esse processo de alinhamento, contribuição principal deste trabalho, temos o enriquecimento prático do tema saindo um pouco das questões filosóficas que o rondam. O processo de alinhamento é apoiado por uma ferramenta implementada em Java que atende a todas as atividades do tipo User Task descritas no processo. Essa ferramenta provê um formulário de entrada para carregamento do mapa estratégico, scripts de transformações escritos em ATL, ambiente visual para interligar (mapear) as dependências dos elementos do CIM2 com as metas do CIM-0, e por fim, verificação de consistência entre os elementos mapeados. Como proposta de trabalho futuro, pode-se analisar a aderência do alinhamento proposto com MDA em processos de outras empresas, em diversos ramos coorporativos.

\section{REFERÊNCIAS}

ATLAS. Atlas Transformation Language. 2012.
Disponível em: <http://iot.eclipse.org > Acesso em: 27 jan. 2015.

BALDAM, R.; VALLE, R.; PEREIRA, H.; ABREU, S. H. ans M.; SOBRAL, V. Gerenciamento de Processos de Negócios: BPM - Business Process Management. São Paulo: Érica, 2007.

BPMN. Business Process Modeling Notation Specification. Needram: Business Process Management Initiative, 2004. 308 p. Acesso em: 9 jun. 2014.

BRODBECK, A. F. Alinhamento Estratégico entre os Planos de Negócio e de Tecnologia de Informação: Um Modelo Operacional para a Implementação. Tese (Doutorado) — UFRGS, 2001.

FERNANDES, A. A.; ABREU, V. F. Implantando a governança de TI: da estratégia à gestão dos processos e serviços. 2. ed. Rio de Janeiro: Brasport, 2008.

FRANKEL, D. S. BPM and MDA. The rise of model-driven enterprise systems. 2003. Disponível em: <http://www.bptrends.com/bpt/wp-content/ publicationfiles/06-03\%20WP\%20BPM\%20and\% 20MDA\%20Whitepaper\%20Frankel11.pdf>.

HARMON, P. Business process change: a manager's guide to improving, redesigning, and automating processes. San Francisco: Morgan Kaufmann Publishers, 2003.

HARRINGTON, H. J.; ESSELING, E. K. C.; NIMWEGEN, H. V. Business Process Improvement: documentation, analysis, design and management of business process improvement. New York: McGraw-Hill, 1997.

HENDERSON, J. C.; VENKATRAMAN, N. Strategic alignment: Leveraging information technology for transforming organizations. IBM System Journal, v. 32, n. 1, p. $4-16,1993$.

JESTON, J.; NELIS, J. Business Process Management: pratical guidelines to successful implementations. Oxford: Elsevier, 2006. P. 299 - 315.

KAPLAN, R. S.; NORTON, D. P. A Estratégia em Ação - Balanced Scorecard. Rio de Janeiro: Elsevier, 1997. 344 p.

Mapas Estratégicos - Convertendo Ativos Intangíveis em Resultados Tangíveis. Rio de Janeiro: Elsevier, 2004. 471 p. 
LAMSWEERDE, A. van; LETIER, E. From object orientation to goal orientation: A paradigm shift for requirements engineering. In: WIRSING, M.; KNAPP, A.; BALSAMO, S. (Ed.). Radical Innovations of Software and Systems Engineering in the Future. Springer Berlin Heidelberg, 2004, (Lecture Notes in Computer Science, v. 2941). p. 325-340. ISBN 978-3-540-21179-2. Disponível em: <http://dx.doi.org/10.1007/978-3-540-24626-8_23>

NIEDERMAN JAMES C. BRANCHEAU, J. C. W. F. Information systems management issues for the 1990s. MIS Quarterly, Management Information Systems Research Center, University of Minnesota, v. 15, n. 4, p. 475-500, 1991. ISSN 02767783. Disponível em: <http://www.jstor.org/stable/249452>

OMG. MDA Guide version 1.0.1. Formal Document: 03-06-01. 2001. Disponível em: <http: //www.omg.org/cgi-bin/apps/doc?omg/03-06-01.pdf> Acesso em: 12 jun. 2014.

REICH, B. H. Investigating the Linkage between Business Objectives and Information Technology Objectives: A multiple case study in the Insureance Industry. Tese (Doutorado) - University of British Columbia, 1992.

ROLLAND, C.; SOUVEYET, C.; ACHOUR, C. Guiding goal modeling using scenarios. Software Engineering, IEEE Transactions on, v. 24, n. 12, p. 1055-1071, Dec 1998. ISSN 0098-5589.

SMITH, H.; FINGAR, P. Business Process Management: The third wave. 1. ed. Tampa: Meghan Kiffer Press, 2003.

VENKATRAMAN, N. IT-Enabled business transformation: From automation to business scope redefinition. Sloan Management Review, p. 73 - 87, 1993.

VOLMER, K. The future of bpm. In: FORRESTER RESEARCH. Anais de The Brainstorm BPM

Conference. Chicago, 2007. 